\title{
On largest Hausdorff compactification for convergence spaces
}

\author{
C.J.M. Rao
}

In this note we obtain a characterization of the class of convergence spaces for which Richardson's compactification is the largest Hausdorff compactification and a characterization of the class of convergence spaces which possess largest Hausdorff compactifications.

It is known that every Hausdorff convergence space [3] and, in particular, every limit space [1] has a Hausdorff compactification. In [5] Richardson obtained a compactification (which he called a Stone-Čech compactification for convergence spaces) for a convergence space $S$ which has the property that every continuous function from $S$ into a regular (regular we mean including Hausdorff) compact convergence space has a unique continuous extension to this compactification. In general, Richardson's compactification is not regular, and therefore need not be the largest regular compactification of $S$. At the same time the regularity requirement on the range space (for extension of continuous functions) can not be in general relaxed, and hence it also fails to be the largest Hausdorff compactification of $S$. Under these circumstances it is natural to ask when is Richardson's compactification the Stone-Čech regular compactification on one hand and when is it Stone-Cech Hausdorff compactification on the other. Recently Gazik [2] has solved the first problem, where he has obtained a characterization of the class of

Received 9 October 1974. The author wishes to thank Professor R.S.L. Srivastava for his encouragement. 
convergence spaces for which Richardson's compactification is regular, and hence is the largest regular compactification. In Section 1 of this note we solve the second problem and obtain a characterization of the class of convergence spaces for which Richardson's compactification is the largest Hausdorff compactification.

Though every Hausdorff convergence space has a Hausdorff compactification, it has been pointed out in [6] that the number of distinct maximal Hausdorff compactifications can be quite large. Therefore, it is of interest to know which convergence spaces have largest Hausdorff compactifications. In Section 2 of this note we obtain a characterization of this class. Interestingly, this class appears to be larger than the class for which Richardson's compactification is largest Hausdorff compactification, and smaller than the class possessing smallest Hausdorff compactifications. We would like to point out that, with the result in this section together with those in [4] and [6], we complete the investigations in extremal regular compactifications and extremal Hausdorf $f$ compactifications of convergence spaces.

For basic definitions about convergence spaces used in this note, we refer to [3]. A convergence space $(S, q)$, where $q$ is the convergence structure, will be denoted simply by $S$, and $q$-convergence points will be referred to as $S$-convergence points. For two convergence spaces $S$, and $S^{\prime}, S \geq S^{\prime}$ will mean the convergence structure of $S$ is finer than the convergence structure of $S^{\prime}$. The same notation $\geq$ will.be used to compare two filters on a set, and two compactifications of a given space, in the usual sense. A subset $S$ of a convergence space $X$ is said to be $X$-open if $S$ belongs to every filter on $X$ which $X$-converges to a point in $S$. A convergence space $S$ is called locally compact if it is open in each of its Heusdorff compactifications. A Hausdorff one point compactification $(T, j)$ for a Hausdorff convergence space $S$ was constructed in [4] and it was show that $S$ has a smallest Hausdorff compactification if and only if $S$ is locally compact. For details of this we refer to [4].

We mention two lemmas, the proof of the first lemme is simple and therefore omitted, and the second lemma was proved implicitly in [4].

LEMMA 1. If $f$ and $g$ are two continuous functions from a 
convergence space $X$ to a Hausdorff convergence space $Y$ such that $f$ and $g$ agree on a dense subset $S$ of $X$, then $f=g$.

LEMMA 2. If $f$ is a continuous function from a Hausdorff convergence space $X$ onto an arbitrary convergence space such that $f$ restricted to a dense subset $S$ of $X$ is isomorphism, then $f(X-S) \cap f(S)=\emptyset$.

Before stating our main theorems we recall some of the notations used in [5]. Let $S$ be a Hausdorff convergence space. Let $X_{1}$ be the set consisting of all $\dot{x}$ for $x$ in $S$ and all non convergent ultrafilters on $S$. Let $i$ be the mapping from $S$ to $X_{1}$ defined as $i(x)=\dot{x}$. Let $F$ be a filter on $S$; then $\hat{F}$ is the filter on $X_{1}$ whose base is $\{\hat{F} \mid F \in F\}$, where $\hat{F}=\left\{H \in X_{1} \mid F \in H\right\}$. A convergence structure is defined on the set $X_{1}$ (and the resulting space is denoted by $X_{1}$ ) as follows; let $\phi \in F\left(X_{1}\right)$, then

$\phi \quad x_{1}$-converges to $\dot{x}$ if and only if $\phi \geq \hat{F}$ for some $F$ $S$-converging to $\boldsymbol{x}$,

$\phi X_{1}$-converges to $v$ if and only if $\phi \geq \hat{v}$, where $v$ is a non convergent ultrafilter on $S$.

The space $\left(x_{1}, i\right)$ is the Richardson's compactification of $s$. For details we refer to [5].

1 .

In this section we obtain a necessary and sufficient condition so that Richardson's compactification $R(S)$ of a Hausdorff convergence space $S$ be the largest Hausdorff compactification.

THEOREM. If $S$ is a Hausdorff convergence space, then Richardson's compactification $\left(X_{1}, i\right)$ of $S$ is the Zargest Hausdorff compactification of $S$ if and only if the following two conditions are satisfied:

(i) $S$ is a locally compact convergence space;

(ii) $\hat{v}=i(v) \wedge \dot{v}$, for every non convergent ultrafilter $v$ on $s$. 
Proof. Let us suppose that $\left(X_{1}, i\right)$ is the largest Hausdorff compactification of $S$. Let $(T, j)$ denote the one point Hausdorff compactification of $S$ constructed in [4]; then $\left(x_{1}, i\right) \geq(T, j)$. Now using Lemma 2 we find $S$ is $X_{1}$-open. Since $\left(X_{1}, i\right)$ is largest, another application of Lemma 2 implies $S$ is open in each of its Hausdorff compactifications, and hence $S$ is locally compact. It should be noted that condition $(i)$ implies that a filter $\phi$ on $X_{1}$ will $x_{1}$-converge to $\dot{x}$ if and only if $i(S) \in \phi$ and $i^{-1}(\phi) \quad S$-converges to $x$. Let us define a new convergence structure on the set $X_{1}$ (and denote the new space by $\left.X_{2}\right)$ as follows; let $\phi \in F\left(X_{1}\right)$, then

$$
\begin{aligned}
& \phi x_{2} \text {-converges to } \dot{x} \text { if and only if } \phi x_{1} \text {-converges to } \dot{x} \text {, } \\
& \phi x_{2} \text {-converges to } v \text { if and only if } \phi \geq \psi \wedge \dot{v} \text {, where } \psi \text { is } \\
& \text { an ultrafilter on } x_{1} \text { which } x_{1} \text {-converges to } v \text {. }
\end{aligned}
$$

It is easy to verify that the space $\left(x_{2}, i\right)$ is a Hausdorff compactification of $S$ and $\left(X_{2}, i\right) \geq\left(X_{1}, i\right)$. Hence by assumption and Lemma 1 , we have $\left(X_{1}, i\right)=\left(x_{2}, i\right)$ and thereby $X_{1}=X_{2}$. This in turn implies $\vartheta \geq \psi \wedge \dot{v}$ for every non convergent ultrafilter $v$ on $S$, where $\psi$ is an ultrafilter on $X_{1}$ which $X_{1}$-converges to $v$. Since $i(v) \geq 0$ and $i(v) \neq \dot{v}$, we have $i(v)=\psi$, implying $\hat{v} \geq i(v) \wedge \dot{v}$. But as also $\dot{v} \geq \hat{v}$ and $i(v) \geq \hat{v}$, we have $\hat{v}=i(v) \wedge \dot{v}$.

To prove that the two conditions are also sufficient, let $(y, k)$ be a Hausdorff compactification of $S$. Let us define a map $f$ from $X_{1}$ to $y$ as follows: $f(\dot{x})=k(x)$ and $f(v)=\operatorname{limk}(v)$. It is easy to see that $f$ is a well defined, onto function and $f \circ i=k$. We will prove that $f$ is continuous, then uniqueness of $f$ will follow from Lemma 1 . Let $\phi \in F\left(X_{1}\right)$ and $\phi X_{1}$-converge to $\dot{x}$; then condition ( $i$ ) inplies that $i(S)$ belongs to $\phi$ and $i^{-1}(\phi)$ S-converges to $x$, and hence $f(\phi)$ $y$-converges to $k(x)=f(\dot{x})$. Let $\phi X_{1}$-converge to $v$; then $\phi \geq \hat{v}$ and hence, by condition (ii), $\phi \geq i(v) \wedge \dot{v}$, and therefore 
$f(\phi) \geq k(v) \wedge f(\dot{v})$, which implies $f(\phi)$ Y-converges to $f(v)$.

2.

In this section we obtain a necessary and sufficient condition so that a Hausdorff convergence space $S$ may have the largest Hausdorff compactification.

THEOREM. A Hausdorff convergence space $S$ has the largest Hausdorff compactification if and only if $S$ has at most finitely many non convergent ultrafilters.

Proof. Let $S$ have only finitely many non convergent ultrafilters. Let us denote by $Y$ the set of all $\dot{x}$ for $x$ in $S$ and all non convergent ultrafilters on $S$. Let $k$ be a function from $S$ to $y$ defined as $k(x)=\dot{x}$. Let us define a convergence structure on $Y$ (and denote the new space by $Y$ ) as follows: let $\phi \in F(Y)$, then

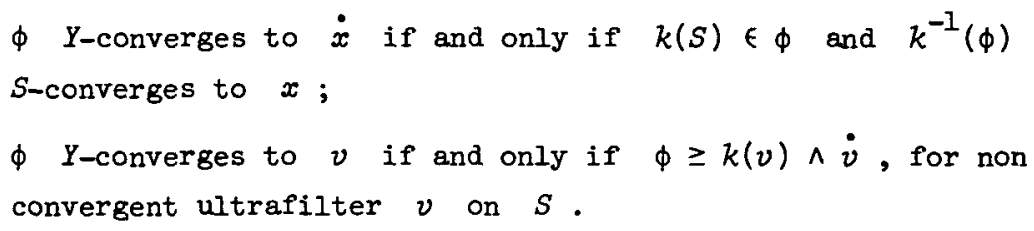

Now it is easy to verify that $(y, k)$ is the largest Hausdorff compactification of $S$.

Conversely, let $(X, f)$ be the largest Hausdorff compactification of $S$. Firstly, this implies $S$ is locally compact. Let $\left(x_{1}, i\right)$ be the Richardson's compactification of $S$. Let $h$ be the continuous function from $x$ onto $X_{1}$ such that $h \circ f=i$. Let us define a map $g$ from $X_{1}$ to $X$ as follows: $g(\dot{x})=f(x)$ and $g(v)=\lim f(v)$ for non convergent ultrafilters $v$ on $S$. Then $g$ is a well defined onto function. Now it is easy to verify that $g \circ h=$ identity on $X$ and $h \circ g=$ identity on $X_{1}$ and hence $g$ is also one to one. Therefore we identify $X$ and $X_{1}$ as sets and take $f$ from $S$ to $X$ as $f(x)=\dot{x}$. Now let us define a new convergence structure on $X$ (and denote the new space by $X^{\prime}$, as follows: let $\phi \in F(X)$, then

$\phi X^{\prime}$-converges to $\dot{x}$ if and only if $\phi X$-converges to $\dot{x}$; 
$\phi \quad X^{\prime}$-converges to $v$ if and only if $\phi \geq \dot{v} \wedge \psi$, where $\psi$ is an ultrafilter on $X$ which $X$-converges to $v$.

Then $\left(X^{\prime}, f\right)$ is a Hausdorff compactification of $S$ and $\left(X^{\prime}, f\right) \geq(X, f)$. And hence by assumption $\left(X^{\prime}, f\right)=(X, f)$ and thereby $X^{\prime}=X$. Next, let $v$ be a non convergent ultrafilter on $S$ and the ultrafilter $f(v)$ on $X$ be $X$-converging to $v^{\prime}$ in $X-f(S)$. Then $h \circ f(v) X_{1}$-converges to $h\left(v^{\prime}\right)$, that is $i(v) X_{1}$-converges to $v^{\prime}$ and therefore $v^{\prime}=v$. This implies $f(v) \geq \psi \wedge \dot{v}$ for some ultrafilter $\psi$ on $X$ which $X$-converges to $v$. Since $f(v) \neq \dot{v}$, we have $f(v)=\psi$. Now, let $\phi$ be an ultrafilter on $X$ containing $X-f(S)$ and let it $X$-converge to $v$ in $X-f(S)$. Then $\phi \geq f(v) \wedge \dot{v}$, since $\phi \neq f(v)$, $\phi=\dot{v}$. Hence each ultrafilter on $X-f(S)$ is a point ultrafilter and hence $X-f(S)$ is finite. Therefore $S$ has at most finitely many non convergent ultrafilters.

The following result is mentioned for its deviation from the topological case.

COROLLARY. If a Hausdorff convergence space has a largest Hausdorff compactification then it also has a smallest Hausdorff compactification.

\section{References}

[1] H.R. Fischer, "Limesräume", Math. Ann. 137 (1959), 269-303.

[2] R.J. Gazik, "Regularity of Richardson's compactification", Canad. J. Math. (to appear).

[3] D.C. Kent, "Convergence quotient maps", Fund. Math. 65 (1969), 197-205.

[4] C.J.M. Rao, "On smallest compactification for convergence spaces", Proc. Amer. Math. Soc. 44 (1974), 225-230.

[5] G.D. Richardson, "A Stone-Čech compactifiçation for limit spaces", Proc. Amer. Math. Soc. 25 (1970), 403-404. 
[6] G.D. Richardson and D.C. Kent, "Regular compactifications of convergence spaces", Proc. Amer. Math. Soc. 31 (1972), 571-573.

Department of Mathematics,

Indian Institute of Technology Kanpur, Kanpur,

India. 